\title{
Cardiac Magnetic Resonance Fingerprinting: Technical Developments and Initial Clinical Validation
}

\author{
G. Cruz $^{1} \cdot$ O. Jaubert ${ }^{1} \cdot$ R. M. Botnar ${ }^{1,2} \cdot$ C. Prieto $^{1,2}$
}

Published online: 27 July 2019

(C) The Author(s) 2019

\begin{abstract}
Purpose of Review Magnetic resonance imaging (MRI) has enabled non-invasive myocardial tissue characterization in a wide range of cardiovascular diseases by quantifying several tissue specific parameters such as $T_{1}, T_{2}$, and $T 2 *$ relaxation times. Simultaneous assessment of these parameters has recently gained interest to potentially improve diagnostic accuracy and enable further understanding of the underlying disease. However, these quantitative maps are usually acquired sequentially and are not necessarily co-registered, making multi-parametric analysis challenging. Magnetic resonance fingerprinting (MRF) has been recently introduced to unify and streamline parametric mapping into a single simultaneous, multi-parametric, fully co-registered, and efficient scan. Feasibility of cardiac MRF has been demonstrated and initial clinical validation studies are ongoing. Provide an overview of the cardiac MRF framework, recent technical developments and initial undergoing clinical validation.

Recent Findings Cardiac MRF has enabled the acquisition of co-registered $T_{1}$ and $T_{2}$ maps in a single, efficient scan. Initial results demonstrate feasibility of cardiac MRF in healthy subjects and small patient cohorts. Current in vivo results show a small bias and comparable precision in $\mathrm{T}_{1}$ and $\mathrm{T}_{2}$ with respect to conventional clinical parametric mapping approaches. This bias may be explained by several confounding factors such as magnetization transfer and field inhomogeneities, which are currently not included in the cardiac MRF model. Initial clinical validation for cardiac MRF has demonstrated good reproducibility in healthy subjects and heart transplant patients, reduced artifacts in inflammatory cardiomyopathy patients and good differentiation between hypertrophic cardiomyopathy and healthy controls.

Summary Cardiac MRF has emerged as a novel technique for simultaneous, multi-parametric, and co-registered mapping of different tissue parameters. Initial efforts have focused on enabling $\mathrm{T}_{1}, \mathrm{~T}_{2}$, and fat quantification; however this approach has the potential of enabling quantification of several other parameters (such as $\mathrm{T}_{2}{ }^{*}$, diffusion, perfusion, and flow) from a single scan. Initial results in healthy subjects and patients are promising, thus further clinical validation is now warranted.
\end{abstract}

Keywords Quantitative MRI · T1 mapping · T2 mapping $\cdot$ Cardiac fingerprinting $\cdot$ Myocardial tissue characterization

\section{Introduction}

In recent years, cardiovascular magnetic resonance (CMR) imaging has emerged as a key modality for non-invasive

This article is part of the Topical Collection on Cardiac PET, CT, and MRI

G. Cruz

gastao.cruz@kcl.ac.uk

O. Jaubert

olivier.jaubert@kcl.ac.uk

R. M. Botnar

rene.botnar@kcl.ac.uk myocardial tissue characterization. In addition to the established late gadolinium enhancement MRI technique, this can be achieved by quantifying several tissue-specific parameters such as $\mathrm{T}_{1}, \mathrm{~T}_{2}$, and $\mathrm{T}_{2}{ }^{*}$ relaxation times, and

\section{Prieto}

claudia.Prieto@kcl.ac.uk

1 School of Biomedical Engineering and Imaging Sciences, King's College London, 3rd Floor, Lambeth Wing, St Thomas' Hospital, London SE1 7EH, UK

2 Pontificia Universidad Católica de Chile Escuela de Ingeniería, Santiago, Chile 
extracellular volume (ECV), enabling detection of focal and diffuse myocardial disease. T1 has been shown to be a sensitive parameter for detection of focal and diffuse fibrosis, whereas $\mathrm{T}_{2}$ and $\mathrm{T}_{2}{ }^{*}$ have been used to quantify edema/inflammation and iron deposition, respectively. CMR parametric mapping is recommended primarily for the assessment of amyloidosis, Anderson-Fabry disease, myocarditis, and myocardial infarction [1]. Moreover, research is currently ongoing to fully validate the performance of these imaging biomarkers in additional diseases such as cardiomyopathies, sarcoidosis, or transplant rejection [2,3]. Simultaneous assessment of these parameters has recently gained interest to potentially improve diagnostic accuracy and enable further understanding of the underlying disease, for example using both $T_{1}$ and $T_{2}$ to differentiate acute/chronic myocardial infarction [4] or to better characterize inflammatory cardiomyopathy [5].

Current clinical methods for cardiac parameter mapping include modified look-locker inversion recovery (MOLLI) [6] for $\mathrm{T}_{1}$ and ECV, $\mathrm{T}_{2}$-prepared balanced steady state-free precession ( $\mathrm{T}_{2}$ prep bSSFP) for $\mathrm{T}_{2}$ [7] and multi-echo gradient echo (MEGE) [8] for $\mathrm{T}_{2}{ }^{*}$. While widely accepted in clinical practice, the accuracy of these methods can vary with sequence parameters and vendors; consequently, acquisition of local reference values is generally recommended. Acquisition of several parameter maps (in multiple slices, pre- and post-contrast agent injection) is generally recommended for myocardial tissue quantification [1]. However, this strategy requires multiple breath-holds, and thus the resulting maps are often not co-registered, hindering analysis and potentially leading to quantification errors. Moreover, among other confounding factors, $T_{2}$ relaxation can affect quantification of $\mathrm{T}_{1}$, and vice versa, if both parameters are not simultaneously included in the signal model.

A new paradigm for unified quantitative parameter mapping, magnetic resonance fingerprinting (MRF), has been recently proposed. In contrast to conventional techniques, MRF provides simultaneous, co-registered, and multiparametric mapping [9] from a single scan, allowing quantification of as many parameters as those included in the signal model. The latter promises not only quantification of several additional parameters of interest (e.g., diffusion, perfusion, CEST) [7], but also the potential correction of confounding factors (e.g., $\mathrm{B}_{0}, \mathrm{~B}_{1}$, magnetization transfer) $[9,10 \bullet \cdot 11]$, that may affect the accuracy, precision and reproducibility (multi-vendor, multi-site) of quantitative cardiac MRI. In MRF, sequence parameters (such as flip angle (FA) and repetition time (TR)) are continuously varied to sensitize the acquisition to tissue parameters of interest (such as $\mathrm{T}_{1}$ and $\mathrm{T}_{2}$ ). Cardiac MRF (cMRF) [10 ••] has been shown to provide simultaneous, co-registered $T_{1}$ and $\mathrm{T}_{2}$ mapping in a single breath-hold. This review will cover the mechanisms of cardiac MRF, recent technical developments, and initial undergoing clinical validation.

\section{Conventional Cardiac Parametric Mapping}

Cardiac $T_{1}$ mapping (pre-contrast native $T_{1}$ or post-contrast $T_{1}$ for ECV) is usually performed with the modified look-locker inversion recovery (MOLLI) [6] type or saturation recovery single-shot acquisition (SASHA) [11] type acquisitions, which employ inversion recovery (IR) or saturation recovery (SR) preparation pulses, respectively. MOLLI uses IR pulses to achieve $T_{1}$ contrast, sampling 8 images with different inversion time delays (TI), acquiring data over 11 heartbeats for a 5(3)3 acquisition; however it is sensitive to heart rate variations, magnetization transfer (MT), and $\mathrm{T}_{2}$ bias [12], among other confounding factors. SASHA relies on SR pulses for $\mathrm{T}_{1}$ encoding, acquiring one image without SR pulse and 9 images following SR pulses with different saturation delays $\left(\mathrm{T}_{1}\right.$ contrasts) in 10 heartbeats. The SR pulse minimizes heart rate dependency and MT effects; however, the reduced dynamic range of the SR vs IR preparation leads to reduced precision in $\mathrm{T}_{1}$ for SASHA. MOLLI is characterized by high precision and a negative bias, whereas SASHA is characterized by high accuracy but lower precision [13].

Cardiac $T_{2}$ mapping is commonly performed with $T_{2}$-prepared balanced steady state-free precession ( $\mathrm{T}_{2}$ prep bSSFP) [7] or $T_{2}$ gradient and spin echo ( $T_{2}$ GraSE) [14] sequences. $\mathrm{T}_{2}$ prep bSSFP achieves $\mathrm{T}_{2}$ contrast by employing $\mathrm{T}_{2}$-prepared modules with different durations, sampling $3 \mathrm{~T}_{2}$-weighted images over 7 heartbeats; however, it is sensitive to the $\mathrm{k}$-space sampling order (linear vs. centric), blood partial volume, and $\mathrm{T}_{1}$ relaxation time [15]. Since the majority of $\mathrm{T}_{2}$ encoding arises from the preparation pulse, other readouts are also suitable for $\mathrm{T}_{2}$-prepared mapping such fast low angle shot (FLASH) [16]. $\mathrm{T}_{2}$ GraSE mapping uses echo planar imaging readouts with varying effective echo times to the $\mathrm{k}$-space center, sampling $9 \mathrm{~T}_{2}$-weighted images in 13 heartbeats; however, it is particularly sensitive to motion, $\mathrm{T}_{2}{ }^{*}$ decay, and signal to noise ratio (SNR) [17].

$\mathrm{T}_{2}{ }^{*}$ mapping is usually performed with multi-echo gradient echo (MEGE) sequences, acquiring $8 \mathrm{~T}_{2}{ }^{*}$-weighted images in a breath-hold; however, this approach is sensitive to magnetic susceptibility, blood partial volume, and SNR [18].

The methods outlined above, while widely accepted in clinical practice, are limited by a variety of confounding factors including inter-parameter dependency of $\mathrm{T}_{1} / \mathrm{T}_{2}{ }^{(*)}$. Moreover, current approaches require multiple acquisitions under several breath-holds to quantify multiple parameters. Notably, all of these methods use steady state sequences that sample discrete points along the $\mathrm{T}_{1} / \mathrm{T}_{2}{ }^{(*)}$ relaxation process, rely mostly on simplified exponential fit models, and map only one parameter at a time. 


\section{Cardiac Magnetic Resonance Fingerprinting}

Instead of sampling a few selected points along the relaxation curves, MRF attempts to capture the continuous transient state of the magnetization history. This is achieved with an acquisition and reconstruction framework that includes three main components: (1) variable pulse sequence sensitized to parameters of interest (e.g., $\mathrm{T}_{1}$ and $\mathrm{T}_{2}$ ); (2) highly undersampled acquisition (enabling high temporal resolution) that introduces incoherent image artifacts; (3) dictionary-based matching for multi-parametric map estimation. Pulse sequences with varying parameters (e.g., FA and TR) are used to generate unique signal evolutions (known as fingerprints) for each tissue, which is described by a given combination of parameters of interest. Highly undersampled k-space trajectories (typically spiral or radial) are employed to sample the transient signal evolution with a high temporal resolution (producing so called time-point images). In contrast to conventional parametric mapping, where a small number ( 10) of fully-sampled (or moderately undersampled) time-point images are acquired, MRF acquires a large number $(\sim 1000)$ of highly undersampled time-point images. Instead of approximated exponential models, MRF uses the Bloch equations (or extended phase graphs $[19,20])$ to simulate all possible tissue-specific signal evolutions for a particular sequence and range of parameters of interest (e.g., $\mathrm{T}_{1} / \mathrm{T}_{2}$ combinations), resulting in a so-called dictionary of fingerprints. Finally, the signal evolution for each pixel in the time-point image series is matched to the closest entry in the dictionary to retrieve the corresponding tissue-specific parameters of interest.

MRF was initially proposed for simultaneous $T_{1}, T_{2}, M_{0}$, and $\mathrm{B}_{0}$ quantification in $2 \mathrm{D}$ brain imaging demonstrating reduced scan times relative to conventional methods. This framework promises several advantages over conventional mapping approaches: unification of multiple (co-registered) parameters in a single scan, natural extension to other parameters of interest (e.g., diffusion or MT) and to model corrections (e.g., $\mathrm{B}_{0}$ or slice profile correction), flexibility in designing optimal MRF sequences for specific applications (e.g., simultaneous $T_{1}$ and $T_{2}$ or simultaneous $T_{2}^{*}$ and diffusion), and higher parametric encoding efficiency, leading to reduced scan times [9, 21-23]. The main drawbacks for MRF are related to the complex acquisition and reconstruction process, which requires advanced sequence design and dictionary simulations to retrieve the underlying parameter values. Regardless, several recent technical developments have extended MRF to map various tissue and system-specific parameters (in addition to $\mathrm{T}_{1}, \mathrm{~T}_{2}$, and $\mathrm{M}_{0}$ ) such as $\mathrm{B}_{0}$ [9], $\mathrm{B}_{1}$ [24], $\mathrm{T}_{2}{ }^{*}$ [25], diffusion [26], fat signal fraction [27], flow [28], and CEST [29].

Cardiac MRF was initially proposed for simultaneous $T_{1}$, $\mathrm{T}_{2}$, and $\mathrm{M}_{0}$ mapping at $3 \mathrm{~T}[10 \bullet \bullet$. Similar to conventional mapping cardiac MRF relies on breath-held acquisitions to minimize respiratory motion, and therefore scan times are limited to achievable breath-hold durations (20-30 s). Electrocardiogram (ECG) triggering is used to minimize cardiac motion, limiting data acquisition to a small window (typically $100-250 \mathrm{~ms}$ ) in the cardiac cycle, usually during middiastole. The need for ECG triggering requires several adaptations for cardiac MRF in comparison with conventional (static, e.g., brain imaging) MRF. In particular, variable magnetization preparation with interleaved IR and $\mathrm{T}_{2}$ prep pulses are employed in cardiac MRF to increase sensitivity to $T_{1}$ and $\mathrm{T}_{2}$ parameters. Moreover, whereas dictionaries can be computed once (per sequence) ahead of time for conventional MRF, cardiac MRF requires subject-specific dictionaries that incorporate information about the heart rate variability ( $R R$ interval) throughout the scan. Parametric maps can be derived from zero-filled reconstructions of highly undersampled timepoint images in conventional MRF. However, in cardiac MRF the amount of acquired data is reduced (due to ECG triggering and breath-hold) and residual aliasing can propagate into errors in the parametric maps. Iterative reconstructions incorporating coil sensitivities (i.e., parallel imaging [30, 31]) and additional constraints (such as sparsity [32] or subspace modeling [33]) have been developed for highly accelerated MRF applications (such as cardiac MRF).

The original cardiac MRF sequence employed a gradient echo readout, acquiring data over 16 heartbeats, divided into 4 blocks of 4 heartbeats each [10*0]. In each block, data is acquired with the following magnetization preparation scheme: IR pulse, no preparation, $\mathrm{T}_{2}$ prep $(40 \mathrm{~ms})$, and $\mathrm{T}_{2}$ prep $(80 \mathrm{~ms})$ (Fig. 1a). Different blocks have different inversion time delays (TI) following the IR pulse $21 \mathrm{~ms}, 100 \mathrm{~ms}, 250 \mathrm{~ms}$, and $400 \mathrm{~ms}$. In each heartbeat, a set of 48 time-point images is acquired with a highly undersampled, golden angle [34], variable density spiral trajectory, leading to an acquisition window of $\sim 250 \mathrm{~ms}$ and a total scan time of $\sim 16 \mathrm{~s}$ (corresponding to 768 total time-point images). FA and TR are varied between approximately $5-15^{\circ}$ and 5-6 ms, respectively. The use of relatively low flip angles help in reducing potential errors from $\mathrm{B}_{1}$ errors. The ECG log containing relative timings of preparation and imaging pulses is recorded during each scan and used to simulate a scan-specific dictionary (Fig. 1b) that accounts for patient-specific heart rate variabilities. $T_{1}$ values in the range 50-5000 $\mathrm{ms}$ and $\mathrm{T}_{2}$ values in the range $6-500 \mathrm{~ms}$ are simulated to generate a dictionary of $\sim 15000$ entries. Time-point images are reconstructed in [35] with an iterative multi-scale algorithm to suppress residual aliasing artifacts. Finally, dictionary-based template matching is applied pixel by pixel to generate $T_{1}, T_{2}$, and $M_{0}$ maps (Fig. 1c).

Cardiac MRF was initially compared with conventional MOLLI and $\mathrm{T}_{2}$ prep bSSFP mapping techniques, demonstrating similar parametric map quality, albeit from a single scan. Cardiac MRF accuracy was similar to MOLLI, whereas a small negative bias was observed when compared with 

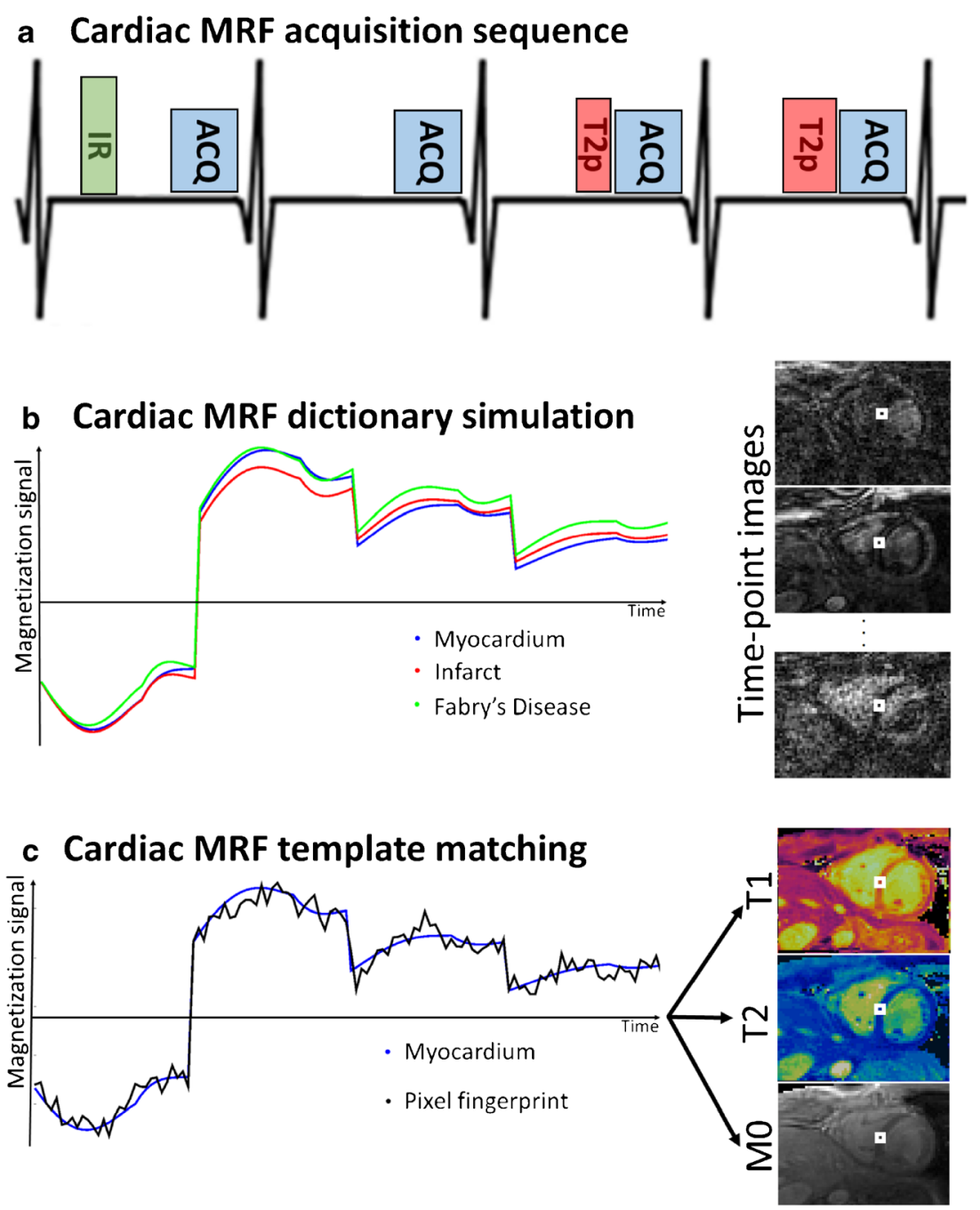

Fig. 1 a Cardiac MRF acquires data with a triggered sequence. In each heartbeat an inversion recovery (IR), $\mathrm{T}_{2}$ preparation (T2p), or no preparation pulse is used before image acquisition (ACQ). This acquisition block is repeated several times during a breath-hold. b Patient-specific information (RR intervals) and sequence information (IR, T2p, FA, TR, etc) are used to simulate a dictionary of all the expected signal evolutions for all tissues of interest (here, three

$\mathrm{T}_{2}$ prep bSSFP; precision for cardiac MRF and conventional methods was similar. Cardiac MRF presented consistent values across varying heart rates (in simulations) and even in reduced number [4-9, 10••, 11, 12] of heartbeats (in vivo) when combined with advanced iterative reconstructions. Initial cardiac MRF results presented some variability in $T_{1}$ and $\mathrm{T}_{2}$ maps due to errors coming from $\mathrm{B}_{0}, \mathrm{~B}_{1}$, slice profile, cardiac motion, residual aliasing, and noise amplification. Additionally, coverage was limited to one slice and only $\mathrm{T}_{1}$ and $\mathrm{T}_{2}$ myocardial quantification was explored.

\section{Cardiac MRF: Technical Developments}

One of the main obstacles for widespread clinical usage of parametric mapping have been confounding factors that affect the representative tissues are shown). The acquired data is reconstructed, producing a large series of time-point images corrupted with undersampling artifacts. c For each pixel in the time-point series, its temporal signal evolution (i.e., fingerprint) is extracted and compared with all the fingerprints in the dictionary via template matching. The highest correlation reveals the underlying tissue and corresponding tissue parameters (i.e., $\mathrm{T}_{1}, \mathrm{~T}_{2}$, and $\mathrm{M}_{0}$ )

accuracy and reproducibility of these techniques. Cardiac MRF is also sensitive to hardware imperfections and undesirable effects from the underlying MR physics. However, cardiac MRF offers a higher flexibility to design sequences that could minimize these errors. Initial research [36 $]$ into confounding factors for cardiac MRF (using gradient echo readouts) has revealed dependencies on slice profile, $\mathrm{B}_{1}$, and efficiency of preparation pulses (IR and $\mathrm{T}_{2}$ prep). Slice profile errors introduce a negative bias in $T_{1}$ and a positive bias in $T_{2}, B_{1}$ errors are closely connected with $T_{2}$ variations and preparation pulse efficiency errors can lead to negative bias in $\mathrm{T}_{1}$ and positive bias in $\mathrm{T}_{2}$. Although these (and more) model corrections can be incorporated into the dictionary simulation, the computational time increases exponentially with the number of parameters. The same study investigated the sensitivity to the above confounding factors for a set of candidate sequences 
with varying combinations of preparation pulses and flip angle patterns. The best performing sequences used varying IR and $\mathrm{T}_{2}$ prep pulses, with flip angles limited to $25^{\circ}$. Considerable errors were observed with high flip angle sequences without any corrections (5-10\% and $4-15 \%$ for $\mathrm{T}_{1}$ and $\mathrm{T}_{2}$, respectively). Errors were reduced (1-2\% and 3-5\% for $\mathrm{T}_{1}$ and $\mathrm{T}_{2}$, respectively) when multiple corrections (for slice profile, $\mathrm{B}_{1}$ and preparation pulse efficiency) were incorporated in the model. Small flip angles $\left(<25^{\circ}\right)$ sequences required less corrections. Optimal sequence design has been studied for MRF [22, 37], but not for ECG-triggered, preparation pulse-based cardiac MRF. Moreover, the investigation of confounding factors in MRF has revealed many effects that can bias results: intra-voxel dephasing [38], incidental spoiler diffusion [39], magnetization transfer [40], $\mathrm{B}_{0}$ [9], $\mathrm{B}_{1}$ [24], slice profile [41, 42], motion [43-45], inversion efficiency [46], partial volume and multi-compartment signal models [47, 48], and residual aliasing artifacts [49, 50].

Multi-slice coverage (basal, mid, and apical) is recommended in myocardial characterization protocols. This has motivated the development of simultaneous multi-slice (SMS) cardiac MRF [51]. SMS uses multiband RF pulses to simultaneously excite several slices with different RF phases; the signal from different slices can then be separated in the reconstruction and template matching steps [52]. SMS was combined with iterative low-rank reconstructions $[33,53]$ to further improve the accuracy and precision of the parameter maps. This approach enabled three slices to be simultaneously acquired in a 16-heartbeat breath-hold. When compared with MOLLI in healthy subjects, SMS cardiac MRF resulted in $\mathrm{T}_{1}$ values approximately $90 \mathrm{~ms}$ higher $(1320 \pm 52 \mathrm{~ms}$ vs. $1242 \pm 33 \mathrm{~ms}$ ), likely due to additional model corrections present in cardiac MRF that are not considered for MOLLI, namely slice profile and relaxation during preparation pulses (leading to imperfect inversions). However, SMS cardiac MRF $T_{1}$ values were still underestimated relative to literature SASHA values, likely due to remaining un-modeled confounding factors such as magnetization transfer [40] or $\mathrm{B}_{1}$ errors [24]. When compared with $\mathrm{T}_{2}$ prep FLASH, SMS cardiac MRF resulted in a negative bias of approximately $6 \mathrm{~ms}(31.7 \pm 1.4 \mathrm{~ms}$ vs. $37.7 \pm 2.3 \mathrm{~ms})$; again this discrepancy is possibly due to remaining confounding factors such as spoiler gradient-induced diffusion [39], magnetization transfer [40], or through-plane motion [43]. A desirable extension for complete myocardial characterization would be 3D whole-heart coverage. Breath-holding is not possible in this case and respiratory motion compensation is required. Preliminary work has shown the feasibility of 3D free-breathing cardiac MRF for whole-heart simultaneous $T_{1}$ and $T_{2}$ mapping [54].

Fat quantification is a useful biomarker to characterize infarction and fatty infiltration [55]; additionally, fatty infiltrations can introduce bias in myocardial tissue characterization [56]. Cardiac MRF has been extended to simultaneous $\mathrm{T}_{1}, \mathrm{~T}_{2}$, and fat fraction quantification [57] at 1.5T. To achieve this, a 3-point Dixon [58, 59] acquisition is incorporated into the cardiac MRF framework to enable water/fat separation. Instead of spiral sampling, a radial trajectory is used to efficiently sample the required Dixon echo times and a local lowrank tensor regularized reconstruction [60] is used to cope with the higher acceleration factors. Dixon cardiac MRF was compared with MOLLI and SASHA in healthy subjects, demonstrating $\mathrm{T}_{1}$ values of $1033 \pm 51 \mathrm{~ms}, 1020 \pm 66 \mathrm{~ms}$, and 1126 $\pm 121 \mathrm{~ms}$, respectively; for $\mathrm{T}_{2}$, Dixon cardiac MRF and $\mathrm{T}_{2}$ GraSE values were $43.2 \pm 4.4 \mathrm{~ms}$ and $51.2 \pm 4.9 \mathrm{~ms}$, respectively; finally a fat fraction of $8 \%$ was measured in healthy subjects with this method. An alternative cardiac MRF approach for simultaneous $T_{1}, T_{2}$, and water/fat separation has also been developed using a Rosette trajectory [61]. This trajectory crosses the k-space center several times during readout. Fat or water suppression can be achieved by demodulating the acquired k-space data by the water or fat precession frequencies, respectively. Preliminary results from Rosette cardiac MRF (at $3 \mathrm{~T}$ ) produced $\mathrm{T}_{1}$ and $\mathrm{T}_{2}$ values of $1329 \pm 22 \mathrm{~ms}$ and $30.7 \pm 2.1 \mathrm{~ms}$, respectively, in addition to water and fat separated proton density maps.

Functional CINE MR imaging is a gold standard technique for the visualization of wall motion abnormalities and quantification of ejection fraction. Motion resolved (MORE) cardiac MRF has been proposed [62] in an effort to provide simultaneous $\mathrm{T}_{1}, \mathrm{~T}_{2}$, and CINE information. In contrast to triggered cardiac MRF, in MORE cardiac MRF data is continuously acquired with a radial bSSFP readout and then retrospectively gated into different cardiac phases. IR pulses regularly interrupt the acquisition to provide $T_{1}$ encoding and the variable flip angle bSSFP readout provides $T_{2}$ encoding. This approach produces $T_{1}$ and $T_{2}$ parameter maps in sixteen cardiac phases which could be used for joint tissue characterization and functional assessment. A similar approach, named CINE-MRF [63], also acquires data continuously with a spiral gradient echo readout and retrospectively reconstructs multiple cardiac phases. CINE-MRF estimates the motion within the cardiac cycle via image registration and uses this information to further improve the accuracy and precision of the cardiac resolved $T_{1}$ and $T_{2}$ maps. Twenty-five cardiac phases were obtained in this fashion and single-slice ejection fraction was found in agreement with standard CINE measurements. Another related promising solution for motion resolved parametric mapping is the recently proposed multitasking (MTT) technique [64]. MTT aims to capture all the dynamics of a cardiac MR protocol in a unified reconstruction. Examples of these dynamics include respiratory and cardiac motion, $T_{1}$ and $\mathrm{T}_{2}$ contrast, and contrast perfusion. MTT has been applied for free-breathing, ECG-free $T_{1} / T_{2}$ mapping, acquiring data with a FLASH readout regularly interrupted by hybrid $\mathrm{T}_{2} \mathrm{IR}$ pulses. Respiratory and cardiac motion information is derived from the data itself with this approach and data is reconstructed within a low-rank tensor framework that naturally exploits correlations between different dynamic dimensions. This enables MTT to produce $T_{1}$ and $T_{2}$ maps in any respiratory or cardiac phase. 


\section{Cardiac MRF: Initial Ongoing Clinical Validation}

The accuracy, precision, and reproducibility of cardiac MRF has been validated in 50 healthy subjects at $1.5 \mathrm{~T}$ [65]. Three slices (base, mid, apex) were acquired with cardiac MRF in this study. The mid slice was acquired twice during the same scan session to evaluate reproducibility. Results showed similar values (964 \pm $71 \mathrm{~ms}$ and $978 \pm 33$ for cardiac MRF and MOLLI, respectively; $41.2 \pm 4.2 \mathrm{~ms}$ and $46.6 \pm 2.7 \mathrm{~ms}$ for cardiac MRF and $\mathrm{T}_{2}$ prep bSSFP, respectively) and excellent reproducibility (via the intraclass coefficient) for all methods. Blinded evaluation of the parametric maps revealed a preference for cardiac MRF in terms of sharpness of myocardial anatomy, absence of artifacts, and overall image quality. Inter-site reproducibility of cardiac MRF has also been evaluated in a separate study [66]. Nine healthy subjects were scanned in two different sites using the same 3T scanner model [66]. MOLLI scans were performed at both sites, site A acquired $\mathrm{T}_{2}$ prep bSSFP data whereas site $\mathrm{B}$ acquired $\mathrm{T}_{2}$ prep FLASH data. Excellent reproducibility of cardiac MRF was observed between sites $\left(1354 \pm 40 \mathrm{~ms}\right.$ and $1346 \pm 38 \mathrm{~ms}\left(\mathrm{~T}_{1}\right), 29.7 \pm 2.8 \mathrm{~ms}$ and $29.6 \pm 4.2 \mathrm{~ms}\left(\mathrm{~T}_{2}\right)$, for sites A and B, respectively). Cardiac MRF values were generally higher than MOLLI $(1220 \pm 39 \mathrm{~ms}$ and $1208 \pm 26 \mathrm{~ms}$, for sites A and B) and considerably lower than $\mathrm{T}_{2}$ prep bSSFP and $\mathrm{T}_{2}$ prep FLASH $(41.6 \pm 1.6 \mathrm{~ms}$ and $38.2 \pm$ $2.1 \mathrm{~ms}$, for sites A and B, respectively).

Encouraging results in healthy subject cohorts warranted further evaluation of cardiac MRF in patients. The first reported cardiac MRF clinical application was for heart transplant patients where graft rejection causes edema that can be measured with $T_{2}$. Thirteen patients and five healthy controls were scanned with $2 \mathrm{D}$ cardiac MRF in basal and mid slices; patient biopsies were performed to detect graft rejection [67]. No rejection was observed in patients. Healthy controls showed slightly reduced $T_{1}$ and $T_{2}$ values relative to patients with both cardiac MRF and conventional mapping techniques. Consistent values were obtained in transplant patients: $1252 \pm 53 \mathrm{~ms}$ and $1260 \pm 63 \mathrm{~ms}$ in $\mathrm{T}_{1}$ pre-contrast, $41.7 \pm 4.8 \mathrm{~ms}$ and $40.9 \pm 1.9 \mathrm{~ms}$ in $\mathrm{T}_{2}$ pre-contrast, and $629 \pm$ $91 \mathrm{~ms}$ and $633 \pm 63 \mathrm{~ms}$ in $\mathrm{T}_{1}$ post-contrast, for cardiac MRF and MOLLI/ $/ 2$ prep bSSFP, respectively. More recently, cardiac MRF has been evaluated for inflammatory cardiomyopathy [68] where both $\mathrm{T}_{1}$ (stronger in acute phase) and $\mathrm{T}_{2}$ (stronger in chronic phase) are relevant biomarkers [5]. Furthermore, this disease is common in patients with implantable cardioverter defibrillators that can cause considerable image artifacts. Twenty-four patients with suspected inflammatory cardiomyopathy (4 of them with implants) were scanned with 2D cardiac MRF at 1.5T; no additional model corrections (e.g., slice profile and inversion efficiency) were used. Cardiac MRF $\mathrm{T}_{1}$ values were similar to MOLLI $\left(1028 \pm 64 \mathrm{~ms}\right.$ and $1019 \pm 53 \mathrm{~ms}$, respectively) whereas $T_{2}$ values were slightly higher than $\mathrm{T}_{2}$ prep bSSFP $(52.8 \pm 3.8 \mathrm{~ms}$ and $49.3 \pm$ $3.1 \mathrm{~ms}$, respectively). Images from patients with implants were scored on the presence of artifacts in the left ventricle (on the scale $0-4,4$ corresponding to no apparent artifacts), revealing a preference for cardiac MRF in both $\mathrm{T}_{1}(3.0 \pm 0.8$ vs. $2.3 \pm 1.0)$ and $\mathrm{T}_{2}(2.8 \pm 1.0$ vs. $1.5 \pm 1.0)$ parameter maps with respect to conventional MOLLI and T2prep bSSFP. Cardiac MRF has recently been applied in patients with hypertrophic cardiomyopathy (HCM) in two studies at 1.5T [69] and 3T [70]. The study at $1.5 \mathrm{~T}$ compared 2D cardiac MRF with MOLLI and $\mathrm{T}_{2}$ prep bSSFP, preand post-contrast, in 6 patients and 12 healthy subjects (without extra model corrections for cardiac MRF). Despite some differences between cardiac MRF and conventional methods, both approaches found significant differences between healthy subjects and HCM patients for pre-contrast $T_{1}$ (cardiac MRF $921 \pm 65 \mathrm{~ms}$ and $1017 \pm 31 \mathrm{~ms}$, respectively; MOLLI $996 \pm 23 \mathrm{~ms}$ and $1057 \pm$ $76 \mathrm{~ms}$, respectively) and ECV (cardiac MRF $25 \pm 3 \%$ and $37 \pm$ $4 \%$, respectively; MOLLI $21 \pm 2 \%$ and $32 \pm 2 \%$, respectively). No significant differences between healthy subjects and HCM patients were observed in pre-contrast $\mathrm{T}_{2}$ (cardiac MRF $43.7 \pm$ $4.3 \mathrm{~ms}$ and $45.0 \pm 5.8 \mathrm{~ms}$, respectively; $\mathrm{T}_{2}$ prep bSSFP $43.7 \pm$ $1.8 \mathrm{~ms}$ and $45.1 \pm 3.7 \mathrm{~ms}$, respectively). Interestingly, significant differences between healthy subjects and HCM patients (32.1 \pm $2.6 \mathrm{~ms}$ and $35.9 \pm 3.2 \mathrm{~ms}$, respectively) were also observed for post-contrast $\mathrm{T}_{2}$ with cardiac MRF. The study at $3 \mathrm{~T}$ compared cardiac MRF with MOLLI and $\mathrm{T}_{2}$ prep FLASH, pre- and postcontrast, in $23 \mathrm{HCM}$ patients. General image quality and artifact presence was evaluated in 1-5 Likert scale (5 corresponding to excellent quality). This analysis revealed a preference for conventional mapping methods pre-contrast and similar quality post-contrast. Cardiac MRF $T_{1}$ values were higher than MOLLI pre$(1397 \pm 40 \mathrm{~ms}$ vs. $1252 \pm 25 \mathrm{~ms})$ and post- $(521 \pm 46 \mathrm{~ms}$ vs. $488 \pm 36 \mathrm{~ms}$ ) contrast; significant differences were also observed for cardiac MRF and MOLLI derived ECV $(27.6 \pm 2.7 \%$ vs. 23.4 $\pm 2.0 \%$, respectively). Cardiac MRF $\mathrm{T}_{2}$ values were also considerably lower than $\mathrm{T}_{2}$ prep FLASH $(29.0 \pm 2.7 \mathrm{~ms}$ vs. $39.7 \pm$ $1.7 \mathrm{~ms}$, respectively). A summary of the parameter values determined in these studies is compiled in Table 1.

\section{Summary and Future Directions}

Cardiac magnetic resonance fingerprinting has been recently introduced, enabling simultaneous and co-registered $T_{1}$ and $\mathrm{T}_{2}$ mapping in a single breath-hold. Initial studies in healthy subjects and patients have shown that cardiac MRF achieves comparable parametric map quality to conventional methods (in reduced scan time); however, several biases have been detected. Reduction of bias due to sequences (e.g., MOLLI vs SASHA, $\mathrm{T}_{2}$ prep bSSFP vs $\mathrm{T}_{2}$ GraSE), vendors and confounding factors has been an important research focus over the last decade to achieve truly quantitative cardiac MR imaging. MRF could contribute to this goal due to its flexibility to incorporate model corrections, which already has been shown to remove some of these confounding factors. In theory, all known factors from the subject's physiology, MR physics and hardware could be incorporated into the cardiac MRF framework. In practice, this would lead to infeasible dictionary 


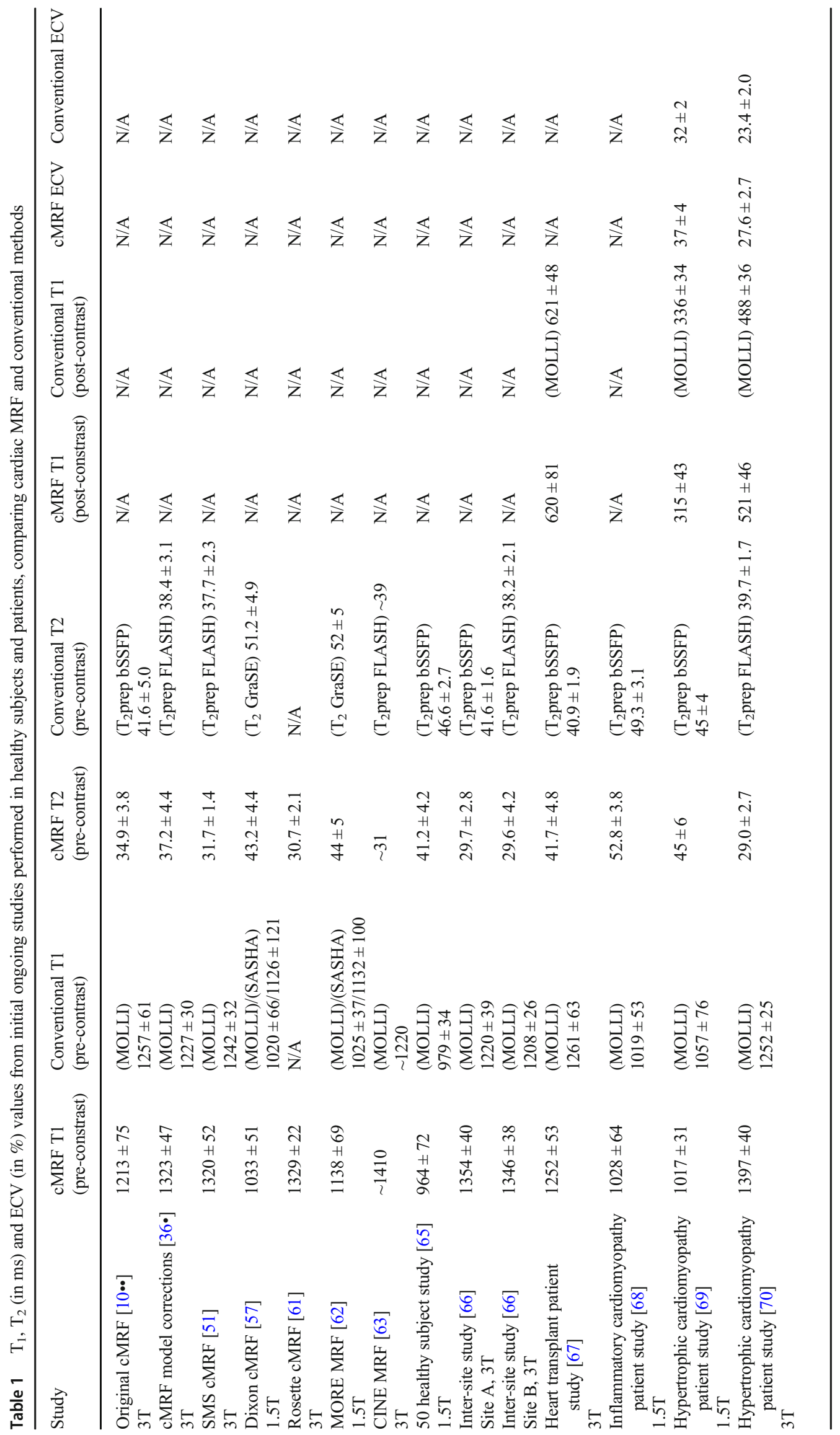


computation times; however, recent advances in machine learning for Bloch simulations could dramatically reduce computational requirements [71]. Such developments should also facilitate the extension of current cardiac MRF to additional parameters of interest such as $\mathrm{T}_{2}{ }^{*}$, magnetization transfer, quantitative susceptibility, diffusion, multi-compartment, and perfusion. Multi-parametric analysis (e.g., radiomics) of this new wealth of myocardial tissue information could potentially improve diagnostic accuracy and enable further understanding of the underlying disease. Clinical validation of cardiac MRF is still in its very early stage; however, promising results have been demonstrated in heart transplant patients, and patients with suspected inflammatory and hypertrophic cardiomyopathies. Further clinical validation is now warranted to evaluate the reproducibility of this technique and its potential to eventually replace currently available mapping techniques. Following in-depth clinical validation, cardiac MRF could be clinically available in the next three years. Currently, several technical developments are still needed to fully exploit the potential of cardiac MRF to provide truly quantitative myocardial characterization for a wide range of tissue parameters. Additional validation is required before widespread clinical adoption; however, cardiac MRF holds several promises that may significantly impact the field of quantitative cardiac MRI in the future.

\section{Compliance with Ethical Standards}

Conflict of Interest Gastao Cruz, René M. Botnar, and Claudia Prieto declare that they have no conflict of interest.

Olivier Jaubert's PhD studentship is partially funded by Philips Healthcare.

Human and Animal Rights and Informed Consent This article does not contain any studies with human or animal subjects performed by any of the authors.

Open Access This article is distributed under the terms of the Creative Commons Attribution 4.0 International License (http:// creativecommons.org/licenses/by/4.0/), which permits unrestricted use, distribution, and reproduction in any medium, provided you give appropriate credit to the original author(s) and the source, provide a link to the Creative Commons license, and indicate if changes were made.

\section{References}

Papers of particular interest, published recently, have been highlighted as:

- Of importance

• Of major importance

1. Messroghli DR, Moon JC, Ferreira VM, et al. Clinical recommendations for cardiovascular magnetic resonance mapping of T1, T2, T2* and extracellular volume: a consensus statement by the Society for Cardiovascular Magnetic Resonance (SCMR) endorsed by the
European Association for Cardiovascular. 2017:(1):75.https://doi. org/10.1186/s12968-017-0389-8.

2. Kim PK, Hong YJ, Im DJ, Suh YJ, Park CH, Kim JY, et al. Myocardial T1 and T2 mapping: techniques and clinical applications. Korean J Radiol. 2017;18:113-31. https://doi.org/10.3348/ kjr.2017.18.1.113.

3. Lota AS, Gatehouse PD, Mohiaddin RH. T2 mapping and T2* imaging in heart failure. Heart Fail Rev. 2017;22:431-40. https:// doi.org/10.1007/s10741-017-9616-5.

4. Tahir E, Sinn M, Bohnen S, Avanesov M, Säring D, Stehning C, et al. Acute versus chronic myocardial infarction: diagnostic accuracy of quantitative native $\mathrm{T} 1$ and $\mathrm{T} 2$ mapping versus assessment of edema on standard T2-weighted cardiovascular MR images for differentiation. Radiology. 2017;285:83-91. https://doi.org/10. 1148/radiol.2017162338.

5. Lurz P, Luecke C, Eitel I, Föhrenbach F, Frank C, Grothoff M, et al. Comprehensive cardiac magnetic resonance imaging in patients with suspected myocarditis: The MyoRacer-Trial. J Am Coll Cardiol. 2016;67:1800-11. https://doi.org/10.1016/j.jacc.2016.02. 013.

6. Messroghli DR, Radjenovic A, Kozerke S, Higgins DM, Sivananthan MU, Ridgway JP. Modified look-locker inversion recovery (MOLLI) for high-resolution T 1 mapping of the heart. Magn Reson Med. 2004;52:141-6. https://doi.org/10.1002/mrm. 20110.

7. Huang TY, Liu YJ, Stemmer A, Poncelet BP. T2 measurement of the human myocardium using a $\mathrm{T}$ 2-prepared transient-state trueFISP sequence. Magn Reson Med. 2007;57:960-6. https://doi. org $/ 10.1002 / \mathrm{mrm} .21208$.

8. Westwood M, Anderson LJ, Firmin DN, Gatehouse PD, Charrier $\mathrm{CC}$, Wonke $\mathrm{B}$, et al. A single breath-hold multiecho T2 * cardiovascular magnetic resonance technique for diagnosis of myocardial Iron overload. J Magn Reson Imaging. 2003;39:33-9. https://doi. org/10.1002/jmri.10332.

9. Ma D, Gulani V, Seiberlich N, Liu K, Sunshine JL, Duerk JL, et al. Magnetic resonance fingerprinting. Nature. 2013;495:187-92. https://doi.org/10.1038/nature11971.

10.• Hamilton JI, Jiang Y, Chen Y, et al. MR fingerprinting for rapid quantification of myocardial $\mathrm{T}_{1}, \mathrm{~T}_{2}$, and proton spin density. Magn Reson Med. 2017;77:1446-58. https://doi.org/10.1002/ mrm. 26216 This seminal work provides the technical details of cardiac MR fingerprinting, demonstrating how it can achieve similar performance to standardized methods (e.g. MOLLI, $T_{2} p$ bSSFP) in reduced scan time.

11. Chow K, Flewitt JA, Green JD, Pagano JJ, Friedrich MG, Thompson RB. Saturation recovery single-shot acquisition (SASHA) for myocardial T1mapping. Magn Reson Med. 2014;71:2082-95. https://doi.org/10.1002/mrm.24878.

12. Gai ND, Stehning C, Nacif M, Bluemke DA. Modified look-locker T1 evaluation using Bloch simulations: human and phantom validation. Magn Reson Med. 2013;69:329-36. https://doi.org/10. 1002/mrm.24251.

13. Roujol S, Weingärtner S, Foppa M, Chow K, Kawaji K, Ngo LH, et al. Accuracy, precision, and reproducibility of four T1 mapping sequences: a head-to-head comparison of MOLLI, ShMOLLI, SASHA, and SAPPHIRE. Radiology. 2014;272:683-9. https:// doi.org/10.1148/radiol.14140296.

14. Baeßler B, Schaarschmidt F, Stehning C, Schnackenburg B, Maintz D, Bunck AC. Cardiac T2-mapping using a fast gradient echo spin echo sequence - first in vitro and in vivo experience. $\mathrm{J}$ Cardiovasc Magn Reson. 2015;17:1-8. https://doi.org/10.1186/s12968-0150177-2.

15. Giri S, Chung YC, Merchant A, Mihai G, Rajagopalan S, Raman $\mathrm{SV}$, et al. T2 quantification for improved detection of myocardial edema. J Cardiovasc Magn Reson. 2009;11:1-13. https://doi.org/ 10.1186/1532-429X-11-56. 
16. Frahm J, Haase A. Rapid NMR imaging of dynamic processes using the FLASH technique. Magn Reson Med. 1986;327:321-7.

17. Sprinkart AM, Luetkens JA, Träber F, Doerner J, Gieseke J, Schnackenburg B, et al. Gradient spin echo (GraSE) imaging for fast myocardial T2 mapping. J Cardiovasc Magn Reson. 2015;17: 1-9. https://doi.org/10.1186/s12968-015-0127-z.

18. He T, Gatehouse PD, Anderson LJ, Tanner M, Keegan J, Pennell DJ, et al. Development of a novel optimized breathhold technique for myocardial T2 measurement in thalassemia. J Magn Reson Imaging. 2006;24:580-5. https://doi.org/10.1002/jmri.20681.

19. Jaynes E. Matrix treatment of nuclear induction. Phys Rev. 1955;98:1099-105.

20. Weigel M. Extended phase graphs: dephasing, RF pulses, and echoes - pure and simple. J Magn Reson Imaging. 2015;41:266-95. https://doi.org/10.1002/jmri.24619.

21. Zhao B, Haldar JP, Liao C, Ma D, Jiang Y, Griswold MA, et al. Optimal experiment design for magnetic resonance fingerprinting: Cramér-Rao bound meets spin dynamics. IEEE Trans Med Imaging. 2018;38:1-861. https://doi.org/10.1109/TMI.2018. 2873704.

22. Assländer J, Sodickson DK, Lattanzi R, Cloos MA. Relaxation in polar coordinates : analysis and optimization of MR-fingerprinting. Proc. 25th Sci. Meet. Int. Soc. Magn. Reson. Med. Honolulu, 2017: 127.

23. Leitao D, Hajnal J V, Teixeira RP, Malik S. Parameter encoding efficiency in transient and steady-state quantitative MRI methods. Proc. 27th Sci. Meet. Int. Soc. Magn. Reson. Med. Montr. 2019: 813.

24. Buonincontri G, Sawiak SJ. MR fingerprinting with simultaneous B1 estimation. Magn Reson Med. 2016;76:1127-35. https://doi. org/10.1002/mrm.26009.

25. Han D, Hong T, Kim D. Development of magnetic resonance fingerprinting (MRF) combined with FISP and multi- echo SPGR acquisition for proton density, T1, T2, T2* and field mapping . Proc. 25th Sci. Meet Int Soc Magn Reson Med Honolulu 2017: 3711.

26. Jiang Y, Hamilton JI, Lo W, et al. Simultaneous T1, T2 and diffusion quantification using multiple contrast prepared magnetic resonance fingerprinting. Proc. 25th Sci. Meet. Int. Soc. Magn. Reson. Med. Honolulu 2017:1171 https://doi.org/10.1002/mrm.25559.7.

27. Ostenson J, Damon BM, Welch EB. MR fingerprinting with simultaneous $\mathrm{T} 1, \mathrm{~T} 2$, and fat signal fraction estimation with integrated B 0 correction reduces bias in water T 1 and T 2 estimates. Magn Reson Imaging. 2019;60:7-19. https://doi.org/10.1016/j.mri.2019. 03.017.

28. Flassbeck S, Schmitter S, Schmidt S, Bachert P, Ladd ME. Flow MR fingerprinting. Magn Reson Med. 2019;81:2536-50. https:// doi.org/10.1002/mrm.27588.

29. Cohen O, Huang S, Mcmahon MT, Rosen MS, Farrar CT. Rapid and quantitative chemical exchange saturation transfer ( CEST ) imaging with magnetic resonance fingerprinting ( MRF ). Magn Reson Med. 2018;80:2449-63. https://doi.org/10.1002/mrm. 27221.

30. Pruessmann KP, Weiger M, Bo P, Boesiger P. Advances in sensitivity encoding with arbitrary $\mathrm{k}$-space trajectories. Magn Reson Med. 2001;651:638-51.

31. Griswold MA, Jakob PM, Heidemann RM, Nittka M, Jellus V, Wang J, et al. Generalized autocalibrating partially parallel acquisitions (GRAPPA). Magn Reson Med. 2002;1210:1202-10. https:// doi.org/10.1002/mrm.10171.

32. Davies M, Puy G, Vandergheynst P, Wiaux Y. A compressed sensing framework for magnetic resonance fingerprinting. arXiv 2014: 7(4):2623-56.

33. Zhao B, Setsompop K, Adalsteinsson E, Gagoski B, Ye H, Ma D, et al. Improved magnetic resonance fingerprinting reconstruction with low-rank and subspace modeling. Magn Reson Med. 2018;79:933-42. https://doi.org/10.1002/mrm.26701.

34. Winkelmann S, Schaeffter T, Koehler T, Eggers H, Doessel O. An optimal radial profile order based on the golden ratio for timeresolved MRI. IEEE Trans Med Imaging. 2007;26:68-76. https:// doi.org/10.1109/TMI.2006.885337.

35. Pierre EY, Ma D, Chen Y, Badve C, Griswold MA. Multiscale reconstruction for MR fingerprinting. Magn Reson Med. 2016;2492:2481-92. https://doi.org/10.1002/mrm.25776.

36. Hamilton JI, Jiang Y, Ma D, et al. Investigating and reducing the effects of confounding factors for robust T1 and T2 mapping with cardiac MR fingerprinting. Magn Reson Imaging. 2018;53:40-51. https://doi.org/10.1016/j.mri.2018.06.018 Confounding factors are a long standing obsctacle for reproducible quantitative imaging. This work studies some of the confounding factors observed in cardiac MR fingerprinting (e.g. slice profile or $\mathbf{B}_{1}$ corrections) and how these can impact $\mathrm{T} 1$ and $\mathrm{T} 2$ estimation.

37. Zhao B, Haldar JP, Member S, et al. Optimal experiment design for magnetic resonance fingerprinting : cram ' meets spin dynamics. IEEE Trans Med Imaging. 2018;38:1-861. https://doi.org/10.1109/ TMI.2018.2873704.

38. Asslander J, Glaser SJ. Pseudo steady-state free precession for MRfingerprinting. Magn Reson Med. 2016;00:1-11. https://doi.org/10. 1002/mrm.26202.

39. Kobayashi Y, Terada Y. Diffusion-weighting caused by spoiler gradients in the fast imaging with steady-state precession sequence may lead to inaccurate T2 measurements in MR fingerprinting. Magn Reson Med Sci. 2019;18:96-104. https://doi.org/10.2463/ mrms.tn.2018-0027.

40. Hilbert T, Kober T, Zhao T, et al. Mitigating the effect of magnetization transfer in magnetic resonance fingerprinting. Proc. 25th Sci. Meet. Int. Soc. Magn. Reson. Med. Honolulu 2017:74.

41. Hong T, Han D, Kim M, Kim D. RF slice profile effects in magnetic resonance fi ngerprinting. Magn Reson Imaging. 2017;41:73-9. https://doi.org/10.1016/j.mri.2017.04.001.

42. Ma D, Coppo S, Chen Y, McGivney DF, Jiang Y, Pahwa S, et al. Slice profile and $\mathrm{B} 1$ corrections in 2D magnetic resonance fingerprinting. Magn Reson Med. 2017;78:1781-9. https://doi.org/10. $1002 / \mathrm{mrm} .26580$

43. Cruz G, Jaubert O, Schneider T, Botnar RM, Prieto C. Rigid motion - corrected magnetic resonance fingerprinting. Magn Reson Med. 2018;81:947-61. https://doi.org/10.1002/mrm.27448.

44. Bipin B, Dan M, Eric M, Pierre Y, Coppo S, Alan M. Magnetic resonance in medicine image reconstruction algorithm for motion insensitive MR fingerprinting ( MRF ): MORF. Magn Reson Med. 2018;80:2485-500. https://doi.org/10.1002/mrm.27227.

45. Yu Z, Zhao T, Cloos MA, Assländer J, Lattanzi R, Sodickson DK. Exploring the sensitivity of magnetic resonance fi ngerprinting to motion. Magn Reson Imaging. 2018;54:241-8. https://doi.org/10. 1016/j.mri.2018.09.002.

46. Kellman P, Herzka DA, Hansen MS. Adiabatic inversion pulses for myocardial T1-mapping. Magn Reson Med. 2014;71:1428-34. https://doi.org/10.1002/mrm.24793 Adiabatic.

47. Mcgivney D, Deshmane A, Jiang Y, et al. Bayesian estimation of multicomponent relaxation parameters in magnetic resonance fingerprinting. Magn Reson Med. 2018;170:159-70. https://oi.org/ $10.1002 / \mathrm{mrm} .27017$.

48. Tang S, Fernandez-granda C. Multicompartment magnetic resonance fingerprinting. Inverse Probl. 2018;34(9):094005.

49. Doneva M, Amthor T, Koken P, Sommer K, Börnert P. Matrix completion-based reconstruction for undersampled magnetic resonance fingerprinting data. Magn Reson Imaging. 2017;41:41-52. https://doi.org/10.1016/j.mri.2017.02.007.

50. Stolk CC, Sbrizzi A. Understanding the combined effect of $\mathrm{k}$-space undersampling and transient states excitation in MR fingerprinting reconstructions. IEEE Trans Med Imaging. 2019;XX:1-11. 
51. Hamilton JI, Ma D, Chen Y, Seiberlich N. Simultaneous multislice cardiac magnetic resonance fingerprinting using low rank reconstruction. NMR Biomed. 2019;2019:1-16. https://doi.org/10. 1002/nbm.4041.

52. Jiang Y, Ma D, Bhat H, Ye H, Cauley SF, Wald LL, et al. Use of pattern recognition for unaliasing simultaneously acquired slices in simultaneous multislice MR fingerprinting. Magn Reson Med. 2017;1876:1870-6. https://doi.org/10.1002/mrm.26572.

53. Assländer J, Cloos MA, Knoll F, Sodickson DK, Hennig J, Lattanzi R. Low rank alternating direction method of multipliers reconstruction for MR fingerprinting. Magn Reson Med. 2018;79:83-96. https://doi.org/10.1002/mrm.26639.

54. Cruz G, Jaubert O, Schneider T, Bustin A, Botnar RM, Prieto C. Toward 3D free-breathing cardiac magnetic resonance fingerprinting. Proc. 27th Sci. Meet. Int. Soc. Magn. Reson. Med. Montr. 2019:4385. https://doi.org/10.1002/mrm.26639.10.

55. Samanta R, Saurabh K, Chik W, et al. Influence of intramyocardial adipose tissue on the accuracy of endocardial. Circ Arrhythm Electrophysiol. 2017;2017:1-15. https://doi.org/10.1161/CIRCEP. 116.004998 .

56. Kellman P, Bandettini WP, Mancini C, Hammer-Hansen S, Hansen MS, Arai AE. Characterization of myocardial T1-mapping bias caused by intramyocardial fat in inversion recovery and saturation recovery techniques. J Cardiovasc Magn Reson. 2015;17:1-11. https://doi.org/10.1186/s12968-015-0136-y.

57. Jaubert O, Cruz G, Bustin A, Schneider T, Botnar RM, Prieto C. Dixon-cMRF: cardiac tissue characterization using three-point Dixon MR fingerprinting. Proc. 27th Sci. Meet. Int. Soc. Magn. Reson. Med. Montr. 2019:1100. https://doi.org/10.1002/mrm. 26216.2.

58. Dixon WT. Simple proton spectroscopic imaging. Radiology. 1984;153:189-94.

59. Berglund J, Johansson L, Kullberg J. Three-point Dixon method enables whole-body water and fat imaging of obese subjects. Magn Reson Med. 2010;1668:1659-68. https://doi.org/10.1002/mrm. 22385.

60. Bustin A, Cruz G, Jaubert O, Lopez K, Botnar RM, Prieto C. High dimensionality undersampled patch - based reconstruction ( HD PROST ) for accelerated multi - contrast MRI. Magn Reson Med. 2019;81:3705-19. https://doi.org/10.1002/mrm.27694.

61. Liu Y, Hamilton J, Griswold M, Seiberlich N. Fat / water separation and $\mathrm{T} 1$ and $\mathrm{T} 2$ quantification using MRF with a rosette trajectory in the heart and liver. Proc. 27th Sci. Meet. Int. Soc. Magn. Reson. Med. Montr. 2019:4376.

62. Jaubert O, Cruz G, Bustin A, et al. Cardiac motion resolved magnetic resonance fingerprinting with joint reconstruction: jMORE-
MRF. Proc. 27th Sci. Meet. Int Soc Magn Reson Med Montr 2019: 808. https://doi.org/10.1002/mrm.26216.3.

63. Hamilton JI, Griswold M, Seiberlich N. Combined cardiac CINE and T1, T2, and M0 mapping with MR fingerprinting. Proc. 27th Sci. Meet. Int. Soc. Magn. Reson. Med. Montr. 2019:405.

64. Christodoulou AG, Shaw JL, Nguyen C, Yang Q, Xie Y, Wang N, et al. Magnetic resonance multitasking for motion-resolved quantitative cardiovascular imaging. Nat Biomed Eng. 2018;2:215-26. https://doi.org/10.1038/s41551-018-0217-y.

65. Pahwa S, Hamilton J, Adedigba J, et al. Myocardial T1 and T2 mapping using MR fingerprinting: comparison to clinical standards. Proc. 28th Sci. Meet Int Soc Magn Reson Med Paris 2018: 762. https://doi.org/10.1002/mrm.20110.

66. Liu Y, Hopman LHGA, Hamilton J, Hillier E, Friedrich M, Seiberlich N. Inter-site reproducibility of cardiac magnetic resonance fingerprinting T1 and T2 quantification in the ISMRM / NIST MRI system phantom and human heart. Proc. 29th Sci. Meet Int Soc Magn Reson Med Montr 2019:4456.

67. Coristine AJ, Hamilton J, van Heeswijk RB, Hullin R, Seiberlich N. Cardiac magnetic resonance fingerprinting in heart transplant recipients. Proc. 28th Sci. Meet. Int. Soc. Magn. Reson. Med. Paris 2018:675.

68. Vincenti G, Coristine AJ, Hamilton JI, et al. Cardiac magnetic resonance fingerprinting for the investigation of suspected inflammatory cardiomyopathy. Proc. 29th Sci. Meet. Int. Soc. Magn. Reson. Med. Montr. 2019:0781. https://doi.org/10.1002/mrm.26216.

69. Patterson A, Li Y, Cavallo AU, Hamilton J, Gulani V, Seilberlich N. Initial utility of cardiac magnetic resonance fingerprinting for quantitative T1 / T2 parametric mapping in hypertrophic cardiomyopathy. Proc. 29th Sci. Meet. Int. Soc. Magn. Reson. Med. Montr. 2019:2049. https://doi.org/10.1002/mrm.262162.2.

70. Wintersperger BJ, Hamilton J, Houbois C, Liu Y, Hanneman K, Seiberlich N. Quantitative multiparametric myocardial evaluation in hypertrophic cardiomyopathy using cardiac magnetic resonance fingerprinting: comparison to conventional cardiac relaxometry. Proc. 29th Sci. Meet. Int. Soc. Magn. Reson. Med. Montr. 2019: 2022.

71. Hamilton JI, Currey D, Griswold M, Seiberlich N. A neural network for rapid generation of cardiac MR fingeprinting dictionaries with arbitrary heart rhythms. Proc. 29th Sci. Meet. Int. Soc. Magn. Reson. Med. Montr. 2019:2421.

Publisher's Note Springer Nature remains neutral with regard to jurisdictional claims in published maps and institutional affiliations. 